\title{
UNIFORM LOCALIZATION IS ALWAYS UNIFORM
}

\author{
RUI HAN
}

\begin{abstract}
In this note we show that if a family of ergodic Schrödinger operators on $l^{2}\left(\mathbb{Z}^{\gamma}\right)$ with continuous potentials have uniformly localized eigenfunctions then these eigenfunctions must be uniformly localized in a homogeneous sense.
\end{abstract}

\section{INTRODUCTION}

Given a topological space $\Omega$, let $T_{i}: \Omega \rightarrow \Omega$ be commuting homeomorphisms, and let $\mu$ be an ergodic Borel measure on $\Omega$. Let $f: \Omega \rightarrow \mathbb{R}$ be continuous and define $V_{\omega}(n)=f\left(T^{n} \omega\right)$ for $n \in \mathbb{Z}^{\gamma}$, where $T^{n}=T_{1}^{n_{1}} \ldots T_{\gamma}^{n_{\gamma}}$. Let $H_{\omega}$ be the operator on $l^{2}\left(\mathbb{Z}^{\gamma}\right)$,

$$
\left(H_{\omega} u\right)(n)=\sum_{|m-n|=1} u(m)+V_{\omega}(n) u(n) .
$$

The occurrence of pure point spectrum for the operators $\left\{H_{\omega}\right\}$ is called phase stable if it holds for every $\omega \in \Omega$.

For a self-adjoint operator $H$ on $l^{2}\left(\mathbb{Z}^{\gamma}\right)$. We say that $H$ has uniformly localized engenfunctions $(U L E)$, if $H$ has a complete set of orthonormal eigenfunctions $\left\{\phi_{n}\right\}_{n=1}^{\infty}$, and there are $\alpha>0, C>0$, such that

$$
\left|\phi_{n}(m)\right| \leq C e^{-\alpha\left|m-m_{n}\right|}
$$

for all eigenfunctions $\phi_{n}$ and suitable $m_{n}$. It is known that $U L E$ has a close connection with phase stability of pure point spectrum. Actually in paper [4, Jitomirskaya pointed out that instability of pure point spectrum implies absence of uniform localization. It is also shown in [3] that if $H_{\omega}$ has $U L E$ for $\omega$ in a set of positive $\mu$-measure, then $H_{\omega}$ has pure point spectrum for any $\omega \in \operatorname{supp}(\mu)$. The proof of this statement mainly relies on the fact that $U L E$ implies uniform dynamical localization $(U D L)$, which means if $H_{\omega}$ has $U L E$, then

$$
\left|\left(\delta_{l}, e^{-i t H_{\omega}} \delta_{m}\right)\right| \leq C_{\omega} e^{-\alpha_{\omega}|l-m|}
$$

for some constants $\alpha_{\omega}$, and $C_{\omega}$ that depend on $\omega$. Recently in [1] and [2, Damanik and Gan established $U L E$ for a certain model and then proved that for this model, actually $C_{\omega}$ and $\alpha_{\omega}$ can be chosen to be independent of $\omega$. In this note we will show that the latter property is always a corollary of $U L E$.

First, let us give a new definition.

Definition 1.1. $H_{\omega}$ has uniform or homogeneous $U L E$ in a set $S$ means that $H_{\omega}$ has $U L E$ for any $\omega$ in $S$ and

$$
\left|\phi_{n}^{\omega}(m)\right| \leq C e^{-\alpha\left|m-m_{n}^{\omega}\right|}
$$

with constants $\alpha>0$ and $C>0$ which do not depend on $\omega$.

Then the main theorems in the note can be stated as follows:

\footnotetext{
${ }^{\dagger}$ This work was partially supported in part by DMS-1401204.
} 
Theorem 1.1. If $H_{\omega}$ has $U L E$ for $\omega$ in a positive $\mu$-measure set, then $H_{\omega}$ has homogeneous $U L E$ in $\operatorname{supp}(\mu)$.

Theorem 1.1. If $T$ is minimal, and $H_{\omega}$ has $U L E$ at a single $\omega$, then $H_{\omega}$ has homogenous $U L E$ in $\Omega$.

\section{Proof of Theorem 1.1}

Let $\left\{\mathbf{U}_{k}\right\}$ be a family of transitions on $l^{2}\left(\mathbb{Z}^{\gamma}\right)$ defined by $\left(\mathbf{U}_{k} u\right)(m):=u(m-k)$. Clearly, if $\left\{\phi_{n}^{\omega}\right\}$ is a complete set of eigenfunctions of $H_{\omega}$, then $\left\{\mathbf{U}_{k} \phi_{n}^{\omega}\right\}$ is a complete set of eigenfunctions of $H_{T^{k} \omega}$. Also, if $H_{\omega}$ has $U L E$, which means $\exists \alpha_{0}>0, C_{0}>0$ such that

$$
\left|\phi_{n}^{\omega}(m)\right| \leq C_{0} e^{-\alpha_{0}\left|m-m_{n}^{\omega}\right|}
$$

for all eigenfunctions $\phi_{n}^{\omega}$ and suitable $m_{n}^{\omega}$. Then $H_{T^{k} \omega}$ also has $U L E$. In fact if we let $m_{n}^{T^{k} \omega}=m_{n}^{\omega}+k$, then

$$
\left|\phi_{n}^{T^{k} \omega}(m)\right|=\left|\left(\mathbf{U}_{k} \phi_{n}^{\omega}\right)(m)\right| \leq C_{0} e^{-\alpha_{0}\left|m-m_{n}^{T^{k} \omega}\right|}
$$

for all eigenfunctions $\mathbf{U}_{k} \phi_{n}^{\omega}$, also notice that the constants $C_{0}$ and $\alpha_{0}$ are the same for $H_{\omega}$ and $H_{T^{k} \omega}$.

Lemma 2.1. If $H_{\omega}$ has $U L E$ for $\omega$ in a positive $\mu$-measure set $S$, then $H_{\omega}$ has $U L E$ for a.e. $\omega \in \Omega$.

Proof. $H_{\omega}$ has $U L E$ in $\bigcup_{k \in \mathbb{Z}^{\gamma}} T^{k} S$, which is a transition invariant set, so $\mu\left(\Omega \backslash\left(\bigcup_{k \in \mathbb{Z}^{\gamma}} T^{k} S\right)\right)=$ 0 .

Theorem 2.2. If $H_{\omega}$ has $U L E$ for a.e. $\omega \in \Omega$, then $\exists \alpha>0$ independent of $\omega$, such that

$$
\left|\phi_{n}^{\omega}(m)\right| \leq C_{\omega} e^{-\alpha\left|m-m_{n}^{\omega}\right|}
$$

for a.e. $\omega \in \Omega$ and all eigenfunctions $\phi_{n}^{\omega}$ with suitable $m_{n}^{\omega}$.

Proof. Let $\bigcup_{j=1}^{\infty}\left\{\omega|| \phi_{n}^{\omega}(m) \mid \leq C_{\omega} e^{-\frac{1}{j}\left|m-m_{n}^{\omega}\right|}\right.$, for all eigenfunctions $\phi_{n}^{\omega}$ and suitable $\left.m_{n}^{\omega}\right\}:=$ $\bigcup_{j=1}^{\infty} A_{j} . \quad A_{j}$ is translation invariant. Since $\mu\left(\bigcup_{j=1}^{\infty} A_{j}\right)=1, \exists j_{0}$ such that $\mu\left(A_{j_{0}}\right)=1$.

Now, let's return to the proof of Theorem 1.1.

Proof of Theorem 1.1. By Theorem 2.2, and direct computation we have $\left|\left(\delta_{l}, e^{-i t H_{\omega}} \delta_{m}\right)\right| \leq$ $C_{\omega} e^{-\alpha|l-m|}$ for a.e. $\omega$. Let

$$
F(\omega)=\sup _{t \in \mathbb{Q}, l, m \in \mathbb{Z} \gamma}\left|\left(\delta_{l}, e^{-i t H_{\omega}} \delta_{m}\right)\right| e^{\alpha|l-m|} .
$$

Then $F(\omega)<\infty$ a.e. $\omega$. It is also easy to see that $F(\omega)$ is measurable and translation invariant. Therefore, by ergodicity, $F(\omega)=C$ a.e. $\omega$. Hence $\left|\left(\delta_{l}, e^{-i t H_{\omega}} \delta_{m}\right)\right| \leq C e^{-\alpha|l-m|}$ a.e. $\omega$. Then on a dense set in $\operatorname{supp}(\mu)$,

$$
\left|\left(\delta_{l}, e^{-i t H_{\omega}} \delta_{m}\right)\right| \leq C e^{-\alpha|l-m|} .
$$

By continuity, the inequality holds for any $\omega$ in $\operatorname{supp}(\mu)$.

Then since $P_{\{E\}}^{\omega}={ }_{\text {S- }} \lim _{T \rightarrow \infty} \frac{1}{2 T} \int_{-T}^{T} e^{i E s} e^{-i H_{\omega} s} d s$, we have

$$
\left|\left(\delta_{l}, P_{\{E\}}^{\omega} \delta_{m}\right)\right| \leq C e^{-\alpha|l-m|}
$$


for any $E \in \mathbb{R}$. Therefore if we choose $\tilde{m}_{n}^{\omega}$ so that $\left|\phi_{n}^{\omega}\left(\tilde{m}_{n}^{\omega}\right)\right|=\sup _{m}\left|\phi_{n}^{\omega}(m)\right|$, we get

$$
\left|\phi_{n}^{\omega}(l)\right|^{2} \leq\left|\phi_{n}^{\omega}(l)\right|\left|\phi_{n}^{\omega}\left(\tilde{m}_{n}\right)\right| \leq C e^{-\alpha\left|l-\tilde{m}_{n}^{\omega}\right|}
$$

Remark 2.1. For the proof of Theorem $1.1^{\prime}$, one need to realize that when $T$ is minimal, $F(\omega)$ being translation invariant implies that $F(\omega)$ is constant in a dense subset of $\Omega$.

\section{Generalization}

In fact we can extend the result above to a more general case where $f(x)$ is allowed to have discontinuities.

Definition 3.1. We say $f$ has invariant continuity filter in $\Omega$ if at every $\omega \in \Omega$, there is a filter $F_{\omega}$, such that any $A_{\omega} \in F_{\omega}$ satisfies the following conditions:

1. $\mu\left(A_{\omega} \bigcap B(\omega, \delta)\right)>0$, for any $\delta>0$,

2. $\lim _{\omega_{k} \in A_{\omega}, \omega_{k} \rightarrow \omega} f\left(\omega_{k}\right) \rightarrow f(\omega)$,

3. $T^{n}\left(A_{\omega}\right) \in F_{T^{n} \omega}$, for any $n \in \mathbb{Z}^{\gamma}$.

Example. Let $\Omega=\mathbb{T}=\mathbb{R} / \mathbb{Z}$, and $\mu$ be the Lebesgue measure. For any $\theta \in \Omega, T(\theta)=\theta+\alpha$ where $\alpha \notin \mathbb{Q}$ and $f(x)=\{x\}$. Define $\left(H_{\theta} u\right)(n)=u(n+1)+u(n-1)+f\left(T^{n} \theta\right) u(n)$. The reason why we are interested in this model is that $U L E$ has recently been shown for it in [5]. Obviously in this model $f$ is not continuous but it does have continuity invariant filter at every $\theta \in[0,1]$. In fact, the filter at $\theta$ is the set of all intervals with left endpoint $\theta$. Generally speaking, all the right or left continuous function defined on $\mathbb{R}$ with direction preserving $T$ has invariant continuity filter at every point.

Now we have the following theorem:

Theorem 3.1. Assume $f$ is bounded and has invariant continuity filter at every $\omega \in \Omega$, then if $H_{\omega}$ has $U L E$ in a positive $\mu$-measure set, $H_{\omega}$ has homogenous $U L E$ in $\operatorname{supp}(\mu)$.

As before, we also have:

Theorem 3.1. Assume $f$ is bounded and has invariant continuity filter at every $\omega \in \Omega$, then if $H_{\omega}$ has $U L E$ at a single $\omega, H_{\omega}$ has homogenous $U L E$ in $\Omega$.

Proof. Notice that in the Proof of Theorem 1.1, we only use the continuity of $f$ in the last step, which means we still have

$$
\left(\delta_{l}, e^{-i t H_{\omega}} \delta_{m}\right) \leq C e^{-\alpha|l-m|}
$$

for $\omega \in \Omega_{0}$, where $\mu\left(\Omega_{0}\right)=1$.

Now consider any $\omega_{0} \notin \Omega_{0}$, we know $\mu\left(A_{\omega_{0}} \cap B\left(\omega_{0}, \frac{1}{k}\right)\right)>0$, hence we can choose $\omega_{k}^{(0)} \in$ $A_{\omega_{0}} \cap B\left(\omega_{0}, \frac{1}{k}\right), \omega_{k}^{(0)} \in \Omega_{0}$ and $\left|f\left(\omega_{k}^{(0)}\right)-f\left(\omega_{0}\right)\right|<\frac{1}{2^{k}}$. Then $T^{m} \omega_{k}^{(0)} \in T^{m} A_{\omega_{0}}$, for any $m \in \mathbb{Z}^{\gamma}$. $T^{m} \omega_{k}^{(0)} \rightarrow T^{m} \omega_{0}$, therefore $f\left(T^{m} \omega_{k}^{(0)}\right) \rightarrow f\left(T^{m} \omega_{0}\right)$. Hence we can choose a subsequence of $\left\{\omega_{k}^{(0)}\right\}$, say $\left\{\omega_{k}^{(m)}\right\}$, such that $\left|f\left(T^{m} \omega_{k}^{(m)}\right)-f\left(T^{m} \omega_{0}\right)\right|<\frac{1}{2^{k}}$. Notice that by the diagonal argument, we can find a sequence $\left\{\omega_{k}\right\}$, satisfying $\omega_{k} \in \Omega_{0}$ and $\left|f\left(T^{j} \omega_{k}\right)-f\left(T^{j} \omega_{0}\right)\right|<\frac{1}{2^{k}}$ for any $j \in \mathbb{Z}^{\gamma}$ and $k \geq|j|$.

Now, let us show that for fixed $l, m, t$ :

$$
\left(\delta_{l}, e^{-i t H_{\omega_{k}}} \delta_{m}\right) \rightarrow\left(\delta_{l}, e^{-i t H_{\omega_{0}}} \delta_{m}\right) .
$$


Indeed: $\left|\left(\delta_{l}, e^{-i t H_{\omega_{k}}} \delta_{m}\right)-\left(\delta_{l}, e^{-i t H_{\omega_{0}}} \delta_{m}\right)\right|$

$=\mid\left(\delta_{l},\left(e^{-i t\left(H_{\omega_{k}}-H_{\omega_{0}}\right)}-1\right) e^{\left.-i t H_{\omega_{0}} \delta_{m}\right) \mid}\right.$

$=\left|\left(\delta_{l},\left(e^{-i t\left(H_{\omega_{k}}-H_{\omega_{0}}\right)}-1\right) \sum_{r=-\infty}^{\infty} a_{r} \delta_{r}\right)\right|$

$\leq\left|a_{l}\right|\left(e^{|t|\left|f\left(T^{l} \omega_{k}\right)-f\left(T^{l} \omega_{0}\right)\right|}-1\right) \rightarrow 0$, as $k \rightarrow \infty$.

Hence $\left|\left(\delta_{l}, e^{-i t H_{\omega_{0}}} \delta_{m}\right)\right| \leq C e^{-\alpha|l-m|}$.

\section{ACKNOWLEDGEMENT}

I would like to thank Svetlana Jitomirskaya for her guidance and inspiring discussions on this subject.

\section{REFERENCES}

1. Damanik, D. and Gan, Z., 2010. Limit-periodic Schrdinger operators in the regime of positive Lyapunov exponents. Journal of Functional Analysis, 258(12), pp.4010-4025.

2. Damanik, D. and Gan, Z., 2011. Limit-periodic Schrdinger operators with uniformly localized eigenfunctions. Journal d'Analyse Mathmatique, 115(1), pp.33-49.

3. del Rio, R., Jitomirskaya, S., Last, Y. and Simon, B., 1996. Operators with singular continuous spectrum, IV. Hausdorff dimensions, rank one perturbations, and localization. Journal d'Analyse Mathmatique, 69(1), pp.153-200.

4. Jitomirskaya, S., 1997. Continuous spectrum and uniform localization for ergodic Schrdinger operators. journal of functional analysis, 145(2), pp.312-322.

5. Jitomirskaya, S. and Kachkovskiy, I., 2015. All couplings localization for quasiperiodic operators with Lipschitz monotone potentials. arXiv preprint arXiv:1509.02226.

University of California, Irvine, California

E-mail address: rhan2@uci.edu 